(c) 2008 Elsevier Ltd All rights reserved.

\title{
Rainflow fatigue analysis for loads with multimodal power spectral densities
}

\author{
Michel Olagnon ${ }^{\mathrm{a}, *}$, Zakoua Guédé ${ }^{\mathrm{a}, *}$ \\ ${ }^{a}$ Ifremer Centre de Brest, B.P. 70, F-29280 Plouzané, France \\ *: Corresponding author : Olagnon M. and Guédé Z., email addresses : Michel.Olagnon@ifremer.fr, \\ Zakoua.Guede@ifremer.fr
}

\begin{abstract}
:
This paper provides theoretical formulas to calculate conservative estimates of the rainflow fatigue damage from the spectral parameters for spectra composed of the combination of one or several narrow-band low-frequency loads and a higher frequency one, such as are often encountered with moored marine structures. With respect to the semi-empirical formulas that have been proposed by other authors, those presented here are theoretically constructed from the mathematical definition of rainflow and from the statistical properties of Gaussian processes.
\end{abstract}

Keywords: Fatigue; Spectral fatigue; Rainflow; Random loading

\section{Introduction}

Rainflow counting is the generally accepted and recommended procedure for estimation of fatigue damage of structures submitted to random loading. Rainflow was first defined by Tetsuo Endo (1968) as an algorithm operating on a time-history. Since on many occasions the loads are defined by a power spectrum, Monte-Carlo simulations or the narrow-band approximation and corrective factors thereto are commonly used to estimate rainflow damage. Rychlik (1987) gave a mathematical definition for rainflow, and came with coresearchers during the following decade with a number of theoretical results for Gaussian processes. Among these results, perhaps the two most important ones are on one hand that the narrow-band approximation is always conservative with respect to rainflow counting and on the other hand that a rainflow 
Markov transition probabilities matrix, and thus fatigue damage, can be calculated as soon as the spectral power density is known, with no need for a time-series representation of the load. Unfortunately, though those calculations are well implemented in the WAFO toolbox (Brodtkorb et al. , 2000), they remain complex and may thus not be readily applicable when a very large number of different spectra are to be considered.

As pointed out in the last ISSC Fatigue and Fracture Committee report (ISSC , 2006), in a number of ship and offshore engineering applications loading is the consequence of the combination of several loads pertaining to various frequency ranges, for instance the long-period response of a mooring, quasi-static response to swell and to wind waves, and even possibly natural vibrations at higher frequencies. Most of the time, those components are individually narrow-band, and the damage for each of them can be calculated or conservatively estimated at reasonable computational cost.

However, when considered separately and added (simple summation method), the fatigue damages corresponding to those various frequency components provide a non-conservative estimate of the overall damage. The need appears thus to compute global damage for everyone or a large proportion of the combinations of components that can possibly occur during the whole life of ther investigated structure. Since the corresponding combined spectra can be estimated at low computational costs, damage could be estimated by using the narrow-band approximation on them, but in practice that method is overconservative by a large amount. Simplified formulas that enable to compute global damage from the properties of the individual spectral components are thus of great interest, since going through full computations for millions of loading cases is quite unpractical.

Based on equivalent cycle amplitudes, Lotsberg (2005) proposed a DualNarrow-Band method, yet a similar overestimation of the damage can be observed in many occasions as reported by Huang 86 Moan (2006), and operators may also be faced, for instance for developments in West Africa areas, to spectra that exhibit more than two peaks.

On the basis of the mathematical definition of rainflow, we propose in the following of this paper the derivation of formulas for combining recursively narrow-band low-frequency components to a higher frequency one with reasonable conservatism. Those formulas use a splitting of the rainflow ranges into two sets. For one of them the same p.d.f. as for the high frequency component ranges can be used and appropriate theoretical forms that ensure conservatism are proposed for the p.d.f. of rainflow ranges in the second set. The main advantages of this method are that on one hand as many narrow-band low-frequency components as wanted can be recursively added, and on the other hand that since p.d.f's of the ranges are considered, the method can 
deal with two-slope S-N curves and endurance thresholds.

\section{Assumptions}

Let us consider a signal $s(t)$ that is the sum of a high frequency component $h(t)$ with a spectrum $S_{h}(f)$ of limited but not necessarily narrow bandwidth $\left(S_{h}(f)=0\right.$ for $f<f_{1}$ and for $\left.f>f_{2}\right)$ and of a narrow-bandwidth low frequency component $l(t)$ clearly separate from the high frequency one. Narrowbandwidth is defined as irregularity factor $\eta_{l}$ close to one, i.e. the number of turning points is nearly the same as the number of zero-crossings:

$$
\eta_{l}=\frac{m_{2 l}}{\sqrt{m_{0 l} m_{4 l}}} \approx 1
$$

where $m_{i s}$ is the $i^{\text {th }}$ spectral moment of signal $s$.

Clear frequency separation is defined as:

$$
T_{l}=\sqrt{\frac{m_{0 l}}{m_{2 l}}}>\frac{1}{f_{1}}
$$

and

$$
m_{2 l}<<m_{2 h}
$$

One may note that as a consequence of those conditions,

$$
m_{4 l}<<m_{4 h}
$$

The number of local maxima per time unit in the composite signal is:

$$
N_{s}=\sqrt{\frac{m_{4 l}+m_{4 h}}{m_{2 l}+m_{2 h}}} \approx \sqrt{\frac{m_{4 h}}{m_{2 h}}}=N_{h}=\frac{1}{T_{24 h}}
$$

The composite signal can be defined in terms of the low- and high-frequency components by parameters $\alpha, \zeta$ and $\eta_{h}$, or more simply $\alpha, \beta$ and $\eta_{s}$ as follows: 


$$
\begin{aligned}
\alpha & =\frac{\sqrt{m_{0 h}}}{\sqrt{m_{0 l}+m_{0 h}}} \\
\zeta & =\sqrt{\frac{m_{2 l} m_{0 h}}{m_{0 l} m_{2 h}}}=\frac{T_{02 h}}{T_{02 l}}
\end{aligned}
$$

and thus

$$
\begin{aligned}
m_{0 s} & =\frac{m_{0 h}}{\alpha^{2}}=\frac{m_{0 l}}{1-\alpha^{2}} \\
m_{2 s} & =\frac{\alpha^{2}+\zeta^{2}-\alpha^{2} \zeta^{2}}{\alpha^{2}} m_{2 h}=\frac{\alpha^{2}+\zeta^{2}-\alpha^{2} \zeta^{2}}{\zeta^{2}-\alpha^{2} \zeta^{2}} m_{2 l} \\
\beta & =\frac{N_{l}}{N_{s}} \approx \frac{N_{l}}{N_{h}}=\sqrt{\frac{m_{2 l} m_{2 h}}{m_{4 h} m_{0 l}}}=\eta_{h} \sqrt{\frac{m_{2 l} m_{0 h}}{m_{2 h} m_{0 l}}}=\eta_{h} \zeta \\
\eta_{s} & =\frac{m_{2 s}}{\sqrt{m_{4 s} m_{0 s}}} \\
& =\frac{\alpha^{2}+\zeta^{2}-\alpha^{2} \zeta^{2}}{\alpha^{2}} \sqrt{\frac{\alpha^{2} m_{2 h}^{2}}{m_{0 h} \frac{\alpha^{2}+\left(1-\alpha^{2}\right) \eta_{h}^{2} \zeta^{4}}{\alpha^{2}} m_{4 h}}} \\
& =\frac{\alpha^{2}+\zeta^{2}-\alpha^{2} \zeta^{2}}{\sqrt{\alpha^{2}+\left(1-\alpha^{2}\right) \eta_{h}^{2} \zeta^{4}}} \eta_{h}
\end{aligned}
$$

\section{Rainflow Count}

\subsection{Numbers of cycles}

The rainflow count for the high frequency signal $h(t)$ on duration $\tau$ consists of $N_{h}=\tau \sqrt{\frac{m_{4 h}}{m_{2 h}}}=\frac{\tau}{\eta_{h}} \sqrt{\frac{m_{2 h}}{m_{0 h}}}$ cycles.

The rainflow count for the low frequency signal $l(t)$ consists of $N_{l}=\tau \sqrt{\frac{m_{4 l}}{m_{2 l}}} \approx$ $\tau \sqrt{\frac{m_{2 l}}{m_{0 l}}}$ cycles over that duration.

Given the separation assumptions, $N_{s} \approx N_{h}$, the number of rainflow cycles in the composite signal is approximately equal to that in its high frequency component. An important feature resulting from that approximate equality is that, when considering the combined signal, turning points originally in $l(t)$ will not "point out" between turning points originally in $h(t)$. 


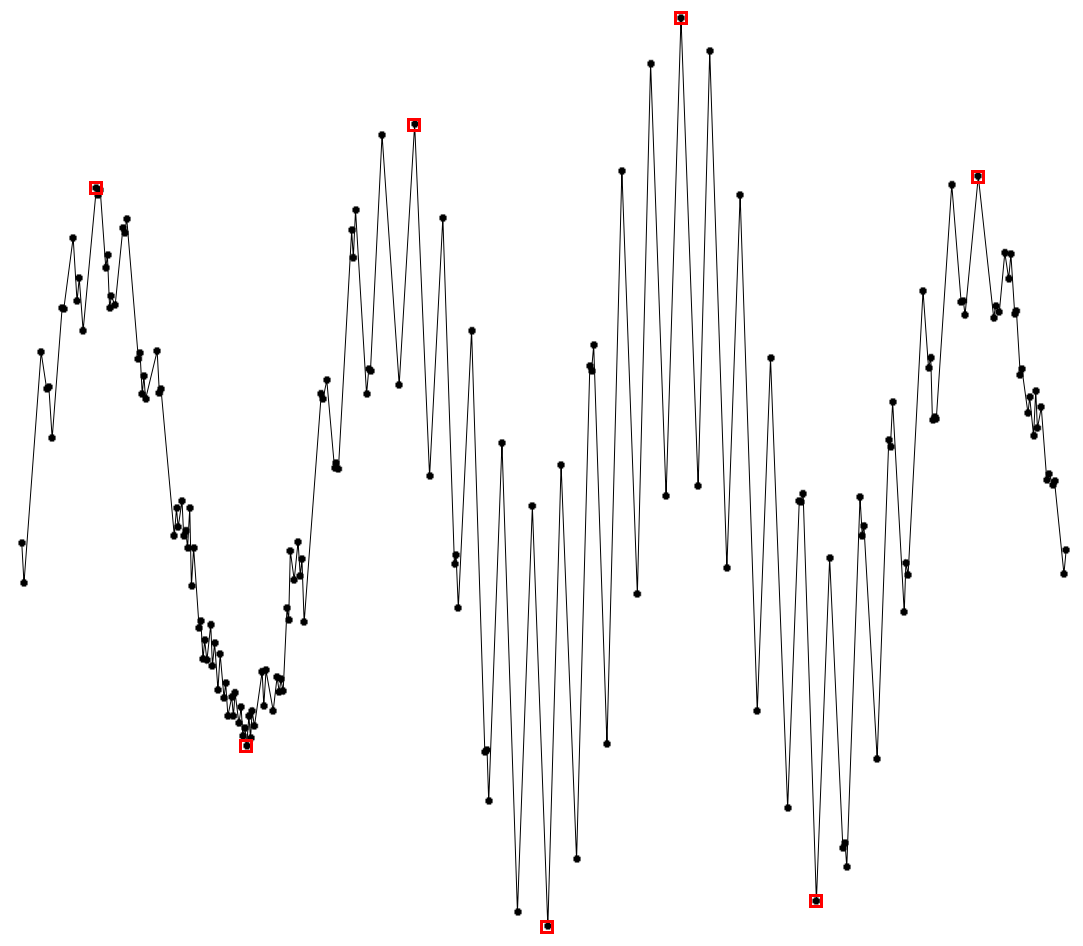

Fig. 1. Subsets $\mathcal{A}$ (dots) and $\mathcal{B}$ (squares)

\subsection{Subsets definition}

We can partition the turning points of signal $s(t)$ into two separate subsets $\mathcal{A}$ and $\mathcal{B}$, shown on figure 1 and defined as follows: 
Let us split time into the consecutive intervals over which $l(t)$ is alternatively positive and negative. A local extremum belongs to $\mathcal{B}$ iif it is the highest turning point of an interval between zero-crossings and where $l(t)$ is positive, or the lowest turning point of an interval where $l(t)$ is negative. The remaining turning points constitute subset $\mathcal{A}$.

In the case where a local maximum in $\mathcal{B}$ would be negative, it can be replaced with a "positive zero" value with a conservative effect since the new sequence is then more damaging, and similarly a positive local minimum yet in $\mathcal{B}$ shall be replaced with a "negative zero".

Using the mathematical definition of rainflow given by Rychlik (1987), it is easy to prove that $\mathcal{B}$ is a "stable" subset in the rainflow count, i.e. that any turning point in $\mathcal{B}$ is associated by the counting procedure over the full signal to another turning point also belonging to $\mathcal{B}$. As a consequence, $\mathcal{A}$ is also rainflow-stable. Turning points in $\mathcal{B}$ will thus contribute $N_{l}$ cycles to the total rainflow count, and turning points in $\mathcal{A}$ the remaining $N_{s}-N_{l} \approx N_{h}-N_{l}$ ones.

\subsection{Ranges associated to subset $\mathcal{A}$}

The number of cycles is reduced according to the removal of turning points belonging to $\mathcal{B}$ :

$$
N_{\mathcal{A}}=N_{s}-N_{l} \approx N_{h}-N_{l}
$$

Let us examine the average amplitudes of the $\mathcal{A}$-ranges. It may be reminded that amplitudes average to the same value for min-max or rainflow counting since that value is the average height of the peaks minus the average height of the troughs, and also twice the average height of the peaks since we are dealing with symetric processes.

Let us denote $M_{s}$ the local maxima of the sum signal and $M_{l}$ (resp. $M_{h}$ ) those belonging to the low-frequency (resp. high-frequency) component.

$E\left(2 M_{s}\right)=2 E\left(M_{h}+l\left(t_{M_{h}}\right)\right)=2 E\left(M_{h}\right)+2 E\left(l(t) \mid t=t_{M_{h}}\right)$ Since the lowand high- frequency components are independent, the average of the lowfrequency signal values at instants of maximal high-frequency components is the same as the global average of the low-frequency signal, i.e. zero and thus the average of the ranges in the sum signal is the same as that of the ranges in the high frequency signal, $2 E\left(M_{s}\right)=2 E\left(M_{h}\right)$. At the same time, one may 
note that mean values in the range mean distribution of $\mathcal{A}$ cycles follow the same distribution as the low-frequency component, i.e. a normal one.

It follows that:

$$
\begin{aligned}
& N_{\mathcal{A}} E\left(M_{A}\right)=N_{s} E\left(M_{h}\right)-N_{\mathcal{B}} E\left(M_{B}\right) \\
& E\left(M_{A}\right)=\frac{N_{s} E\left(M_{h}\right)-N_{l} E\left(M_{B}\right)}{N_{s}-N_{l}} \\
& E\left(M_{A}\right)=E\left(M_{h}\right)\left(1-\frac{\beta}{1-\beta} \frac{E\left(M_{B}\right)-E\left(M_{h}\right)}{E\left(M_{h}\right)}\right)
\end{aligned}
$$

Since the selection process for $\mathcal{B}$ ensures that $E\left(M_{B}\right)>E\left(M_{h}\right)$, the amplitude of ranges in $\mathcal{A}$ is reduced with respect to those of the high-frequency component. Since the selection process only depends of the low-frequency component, that reduction is a global scaling down of the whole distribution when min-max amplitudes are considered.

It may also be noted that two rainflow half-cycles of a signal are either disjunct or contained one in the other. Rainflow half-cycles in subset $\mathcal{A}$ of the sum signal are thus each contained in a half-cycle of subset $\mathcal{B}$, and thus more local (of a lesser time-duration) than those of the high-frequency component. Their distribution is thus closer to the min-max one and less damaging than the rainflow range distribution of the original high-frequency component.

Conservativeness is thus ensured in two ways if the cycle time-density corresponding to subset $\mathcal{A}$ is taken as $(1-\beta)$ that of rainflow cycles of the high-frequency component: conservativeness from the global scaling of the amplitudes and conservativeness from the more local cycles in $\mathcal{A}$.

The use of formula 1 to downscale the distribution remains on the conservative side if $E\left(M_{B}\right)$ is exactly computed or lower bounded.

Figure 2 shows the corresponding distributions in the case of the second application detailed further in this paper.

\subsection{Ranges associated to subset $\mathcal{B}$}

Subset $\mathcal{B}$ contains $N_{l}$ cycles. The maxima and minima in $\mathcal{B}$ are symetrically distributed with respect to 0 , they correspond to a narrow-band (but not Gaussian) process, one may thus consider that the distribution of the rainflow ranges in that process is estimated by that of twice the excursion of the $M_{B}$ points from the mean with reasonnable conservatism. 


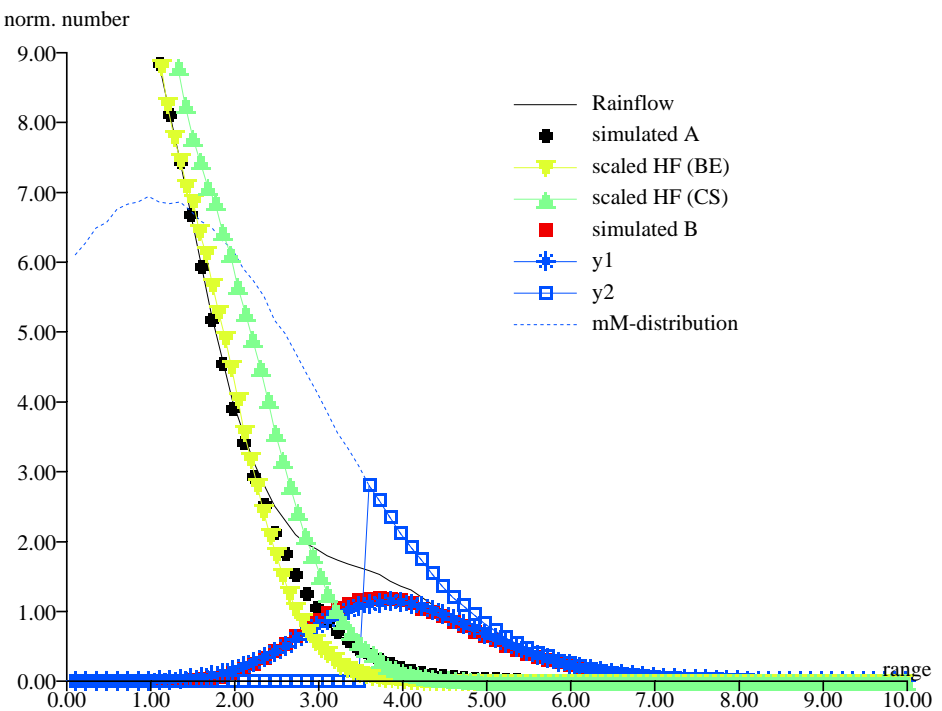

Fig. 2. Distributions of the ranges according to various approximations

The distribution of those $M_{B}$ amplitudes may be estimated in several manners. They are a subset of the positive maxima of the sum signal $s(t)$, and thus the results for the maxima of a gaussian process apply: Let $R$ and $G$ be two independent normalized random variables with Rayleigh and Gaussian 
distribution respectively, then the distribution of the maxima of the random process $s(t)$ is the same as that of the sum

$$
\sqrt{m_{0 s}}\left(\eta_{s} R+\sqrt{1-\eta_{s}^{2}} G\right)=\sqrt{m_{0 s}} M
$$

Finding the distribution $\mathcal{Y}$ of the $M_{B}$ amplitudes corresponds thus to a selection process amongst $\mathcal{M}$-distributed samples, where distribution $\mathcal{M}$ is detailed in appendix $\mathrm{A}$.

\subsubsection{Approximation $\mathcal{Y}_{1}$}

A sensible assumption is that an $M_{B}$ amplitude is the maximum amongst the local maxima that are found between two successive minima of signal $l(t)$. The average number of local maxima in such an interval is $\frac{N_{s}}{N_{l}}$. The correlation between those local maxima is statistically the same for any interval and thus cannot introduce any bias with respect to the distribution of the maximum of the same number of independent local maxima, though the shape of the distribution may be affected. That "correlation" takes the form of a more even spreading of the $\frac{N_{s}}{N_{l}}$ values over the distribution of local maxima than for a subset of independent values, so the correlation should not affect tail of the distribution of the maximum above some threshold.

In that case, the cumulative distribution $\mathcal{Y}_{1}(x)$ for $\frac{M_{B}}{\sqrt{m_{0 s}}}$ can reasonably be derived from the cumulative distribution $\mathcal{M}(x)$ as:

$$
\begin{aligned}
& \mathcal{Y}_{1}(x)=\mathcal{M}^{\frac{N_{s}}{N_{l}}}(x)=\mathcal{M}^{\frac{1}{\beta}}(x) \\
& y_{1}(x)=\frac{1}{\beta} m_{\mathcal{M}}(x) \mathcal{M}^{\frac{1}{\beta}-1}(x)
\end{aligned}
$$

One may note that negative local maxima are in practice forced to zero as described in 3.2 and that they do not contribute to the final rainflow count.

Since $\mathcal{Y}_{1}$ is a "best effort" approximation for the distribution of the $M_{B}$ amplitudes, it is likely that it can be used to provide a good estimate of the rainflow damage due to the $\mathcal{B}$ subset, but conservatism from narrow-band approximation on that subset is not garanteed to compensate for the independence assumption of the local maxima in the case of a very narrow-band $M_{B}$ history and of a low number $\frac{N_{h}}{N_{l}}$. The next approximation $\mathcal{Y}_{2}$ offers a more conservative distribution for the $M_{B}$ amplitudes and may thus be prefered when a conservative estimate is required. 
$\mathcal{Y}_{1}$ can however still be used in that case to estimate the $E\left(M_{B}\right)$ value to apply in formula 1 for the scaling of the ranges in the $\mathcal{A}$ subset.

\subsubsection{Approximation $\mathcal{Y}_{2}$}

A definitely conservative option is to assume that $M_{B}$ amplitudes are the largest possible, i.e. all belong to the upper $\frac{N_{l}}{N_{s}}$ fractile of the global distribution of the maxima of $s(t)$.

$$
\begin{aligned}
\delta_{2} & =\max \left(0, \mathcal{M}^{-1}\left(1-\frac{N_{l}}{N_{s}}\right)\right)=\max \left(0, \mathcal{M}^{-1}(1-\beta)\right) \\
\mathcal{Y}_{2}(x) & =1-\frac{1}{\beta}(1-\mathcal{M}(x)), x \geq \delta_{2} \\
y_{2}(x) & =\frac{m(x)}{\beta}, x \geq \delta_{2}
\end{aligned}
$$

One can see on figure 2 that $y_{1}$ represents very well the actual distribution of the $M_{B}$ peaks, though some slight differences occur at small amplitudes, that we believe to be related to the actual statistical dependence between turning points in the same period of the low-frequency signal. $y_{2}$ is definitely conservative.

\subsubsection{Reference to other authors' approximations}

At this stage, one may note that the Dual Narrow-Band formula proposed by Lotsberg (2005) is a similar case to the above formulas, where $M_{B}$ is assumed to be Rayleigh distributed with a parameter $\sqrt{m_{0 l}}+\sqrt{m_{0 h}}$ equal to the sum of the parameters of the Rayleigh distributions corresponding to the low- and high-frequency signals. In that case,

$$
\mathcal{Y}_{D N B}=\mathcal{R}\left(\frac{\sqrt{m_{0 l}}+\sqrt{m_{0 h}}}{\sqrt{m_{0 l}+m_{0 h}}}\right)=\mathcal{R}\left(\alpha+\sqrt{1-\alpha^{2}}\right)
$$

At the same time, the high frequency component is assumed narrow-band, and thus $\eta_{h}=1$, but the above reasonning shows that such a narrow-band assumption for the high-frequency signal is not necessary.

Similarly, the Jiao $\&$ Moan (1990) Combined Narrow-Band approach also reported in Lie \& Fylling (1994) corresponds to the use of the sum of the low frequency component and of the envelope of the high-frequency one to find 
the distribution of the $M_{B}$ amplitudes. It uses a number of approximations, among which $\alpha^{2}<<1$, and is not garanteed to be conservative.

\section{Miner damage estimates}

Let us consider Miner damage, i.e. following a law of the form

$$
D=\frac{1}{K} \sum_{i=1}^{N} S_{i}^{m}
$$

where $K$ is a material dependent constant and $m$ the slope of the $\mathrm{S}-\mathrm{N}$ curve. Its mathematical expectation may be expressed as:

$$
D=\frac{N}{K} \int_{0}^{\infty} S^{m} p(S)
$$

that is a function of the $m^{\text {th }}$ order moment of the distribution of rainflow ranges. In case a double slope with intersection point at range $\lambda$ is to be considered, the formula becomes an incomplete moment:

$$
D(\lambda)=\frac{N}{K} \int_{\lambda}^{\infty} S^{m} p(S)
$$

and damage can be expressed as:

$$
D=D_{m_{2}}(\lambda)+D_{m_{1}}(0)-D_{m_{1}}(\lambda)
$$

Endurance limits can be handled in a similar manner.

Following the fact that ranges in subset $\mathcal{A}$ can be assumed to have the same distribution as those in the high-frequency signal only, the associated damage can be estimated as the rainflow damage $D_{h}$ associated to that high-frequency signal reduced proportionaly to the reduction in the number of cycles and taking into account the scaling defined by 1 :

$$
\begin{aligned}
& D_{\mathcal{A}}=(1-\beta)\left(1-\frac{\beta}{1-\beta} \frac{E\left(M_{B}\right)-E\left(M_{h}\right)}{E\left(M_{h}\right)}\right)^{m} D_{h} \\
& D_{\mathcal{A}}=(1-\beta)\left(1-\frac{\beta}{1-\beta}(\phi-1)\right)^{m} D_{h}
\end{aligned}
$$


when denoting $\phi=\frac{E\left(M_{B}\right)}{E\left(M_{h}\right)}$. As long as $\phi$ is a lower estimate of the true value, such a reduction is garanteed to remain conservative even when incomplete moments are computed, yet care should be taken that the negative term $-D_{m_{1}}(\lambda)$ in equation 2 needs to be unconservatively estimated since it is substracted from the others.

Subset $\mathcal{B}$ contains $N_{l}$ cycles. Since the low frequency signal is narrow band,

$$
D_{l}=\frac{N_{l}}{K}\left(2 \sqrt{m_{0 l}}\right)^{m} \mu_{m}(\mathcal{R}(1))
$$

where

$$
\mu_{m}(\mathcal{R}(1))=2^{\frac{m}{2}} \Gamma\left(\frac{m}{2}+1\right)
$$

is the $m^{\text {th }}$ moment of a normalized Rayleigh distribution.

Thus,

$$
D_{\mathcal{B}}=\frac{1}{\left(\sqrt{1-\alpha^{2}}\right)^{m}} \frac{E\left(\left(\frac{M_{B}}{\sqrt{m_{0 s}}}\right)^{m}\right)}{\mu_{m}(\mathcal{R}(1))} D_{l}
$$

Denoting $F\left(m, \alpha, \beta, \eta_{s}\right)$ the Rayleigh-normalized moment:

$$
F\left(m, \alpha, \beta, \eta_{s}\right)=\frac{E\left(\left(\frac{M_{B}}{\sqrt{m_{0 s}}}\right)^{m}\right)}{\mu_{m}(\mathcal{R}(1))}
$$

the total damage can be expressed as:

$$
D_{s}=(1-\beta)\left(1-\frac{\beta}{1-\beta}(\phi-1)\right)^{m} D_{h}+\frac{F\left(m, \alpha, \beta, \eta_{s}\right)}{\left(\sqrt{1-\alpha^{2}}\right)^{m}} D_{l}
$$

\subsection{Approximation $\mathcal{Y}_{1}$}

Using function $Z_{1}\left(m, \beta, \eta_{s}\right)$ defined in appendix $\mathrm{B}$, 


$$
\begin{gathered}
F_{1}\left(m, \beta, \eta_{s}\right)=Z_{1}\left(m, \beta, \eta_{s}\right)\left(\sqrt{1-\eta_{s}^{2}}\right)^{m} \\
D_{1 s}=(1-\beta)\left(1-\frac{\beta}{1-\beta}(\phi-1)\right)^{m} D_{h}+Z_{1}\left(m, \beta, \eta_{s}\right)\left(\sqrt{\frac{1-\eta_{s}^{2}}{1-\alpha^{2}}}\right)^{m} D_{l}
\end{gathered}
$$

When $\mathcal{Y}_{1}$ is also used to estimate $\phi$, i.e. $\phi=\frac{\sqrt{1-\eta_{s}^{2}}}{\alpha \eta_{h}} Z_{1}\left(1, \beta, \eta_{s}\right)$, an unconservative estimate of the factor to $D_{h}$ may enfollow. In the following, damage estimated in that manner is subscripted be, as best effort.

Should a conservative estimate be required for each of the $\mathcal{A}$ and $\mathcal{B}$ sets, then we would advise to use the broadband lower-bound for $Z_{1}$, i.e. $\phi=$ $\frac{\sqrt{1-\eta_{s}^{2}}}{\alpha \eta_{h}} Z_{1}(1, \beta, 0)$. In the following, damage estimated in that manner is subscripted $c s$, as conservative scaling.

\subsection{Approximation $\mathcal{Y}_{2}$}

Using the value $\xi_{2}$ and the function $Z_{2}\left(m, \beta, \eta_{s}, x\right)$ defined in appendix B,

$$
\begin{gathered}
F_{2}\left(m, \beta, \eta_{s}\right)=Z_{2}\left(m, \beta, \eta_{s}, \xi_{2}\right)\left(\sqrt{1-\eta_{s}^{2}}\right)^{m} \\
D_{2 s}=(1-\beta)\left(1-\frac{\beta}{1-\beta}(\phi-1)\right)^{m} D_{h}+Z_{2}\left(m, \beta, \eta_{s}, \xi_{2}\right)\left(\sqrt{\frac{1-\eta_{s}^{2}}{1-\alpha^{2}}}\right)^{m} D_{l}
\end{gathered}
$$

Figure 3 shows the damage corresponding to ranges above a given threshold computed with various methods for the second application below and $m=3$. It can be seen that most of the conservatism in $Z_{1}$ approximation is due to the difference between the actual rainflow count in the $\mathcal{B}$ set and the narrow-band approximation. 


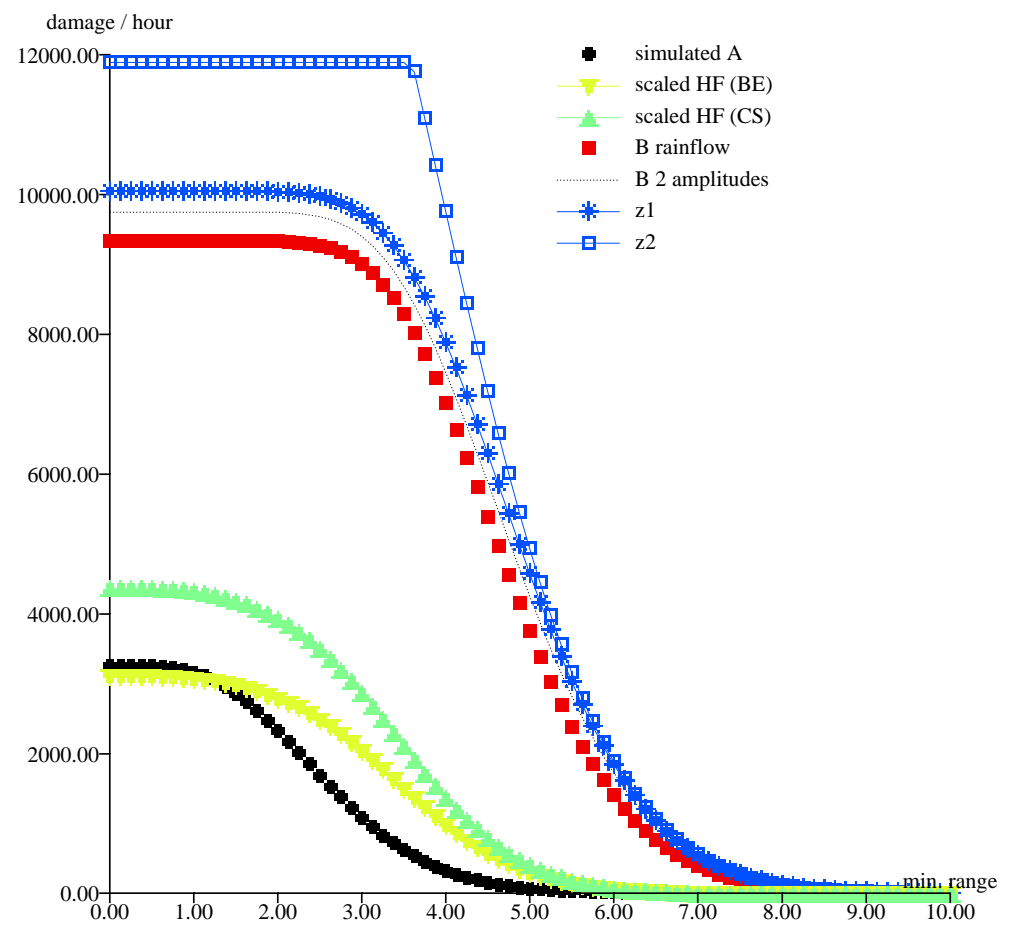

Fig. 3. Comparison of cumulative damage versus range threshold

\subsection{Reference to other authors' approximations}

For the Dual Narrow Band approximation, equation 3 becomes:

$$
D_{D N B s}=(1-\zeta) D_{h}+\left(1+\frac{\alpha}{\sqrt{1-\alpha^{2}}}\right)^{m} D_{l}
$$


For the Jiao Combined Narrow Band approximation, equation 3 becomes:

$$
\begin{aligned}
D_{C N B s}= & D_{h}+\frac{1}{\zeta\left(\sqrt{\left(1-\alpha^{2}\right)}\right)^{m-1}} \sqrt{\left(1-\alpha^{2}\right) \zeta^{2}+\alpha^{2} \delta_{h}^{2}} \\
& \times\left(\left(1-\alpha^{2}\right)^{\frac{m}{2}+1}\left(1-\alpha^{2}-\alpha \sqrt{1-\alpha^{2}}\right)+\alpha \sqrt{\pi} \sqrt{1-\alpha^{2}} \frac{m \Gamma\left(\frac{m+1}{2}\right)}{\Gamma\left(\frac{m}{2}+1\right)}\right) D_{l}
\end{aligned}
$$

where $\delta_{h}$ is the Vanmarcke bandwidth parameter $\sqrt{1-\frac{m_{1}^{2}}{m_{0 h} m_{2 h}}}$ and $\alpha^{2}$ is small.

\section{$5 \quad$ Practical comparisons}

\subsection{Quasi-static response to swell and wind sea}

Let us consider a response spectrum identical to the wave spectrum, composed of a 2.83 meter swell at 15 seconds period, that we model with a triangular spectrum, bandwidth equivalent to Jonswap with $\gamma=19$, and a 2.83 meter wind sea at 5 seconds period that we model with a $\gamma=3$ Jonswap. This spectrum can be seen marqued with dots on figure 4 . The corresponding values of the parameters are: $\alpha=0.7071, \zeta=0.2618, \beta=0.1847, \eta_{s}=0.5157$.

Figure 5 compares damage as computed from the two proposed formulas to a reference obtained by rainflow analysis of the simulation of 2920 3-hour sea states (one year). In addition, a number of other methods are also given in the same table: $D_{S S}=D_{l}+D_{h}$, commonly called simple summation, $D_{D N B}$ using the Dual Narrow Band method, $D_{C N B}$ as defined by Jiao $\&$ Moan (1990) and the narrow-band approximation $D_{N B}$ often called combined spectrum.

In that case, simple summation is definitely unconservative, combined narrow band is slightly more conservative than the narrow-band approximation (combined spectrum) and dual narrow band is overly conservative. As a matter of fact, the spectrum shown in figure 4 is not sufficiently different from a unimodal one for the combined spectrum method to be overly conservative. With the proposed formulas, best effort with $Z_{1}$ results in a good approximation of the rainflow damage though sometimes unconservative, while conservative scaling with $Z_{1}$ provides less than $10 \%$ conservatism. $Z_{2}$ estimates are both conservative at all times, with similar conservatism to the narrow-band approximation. 


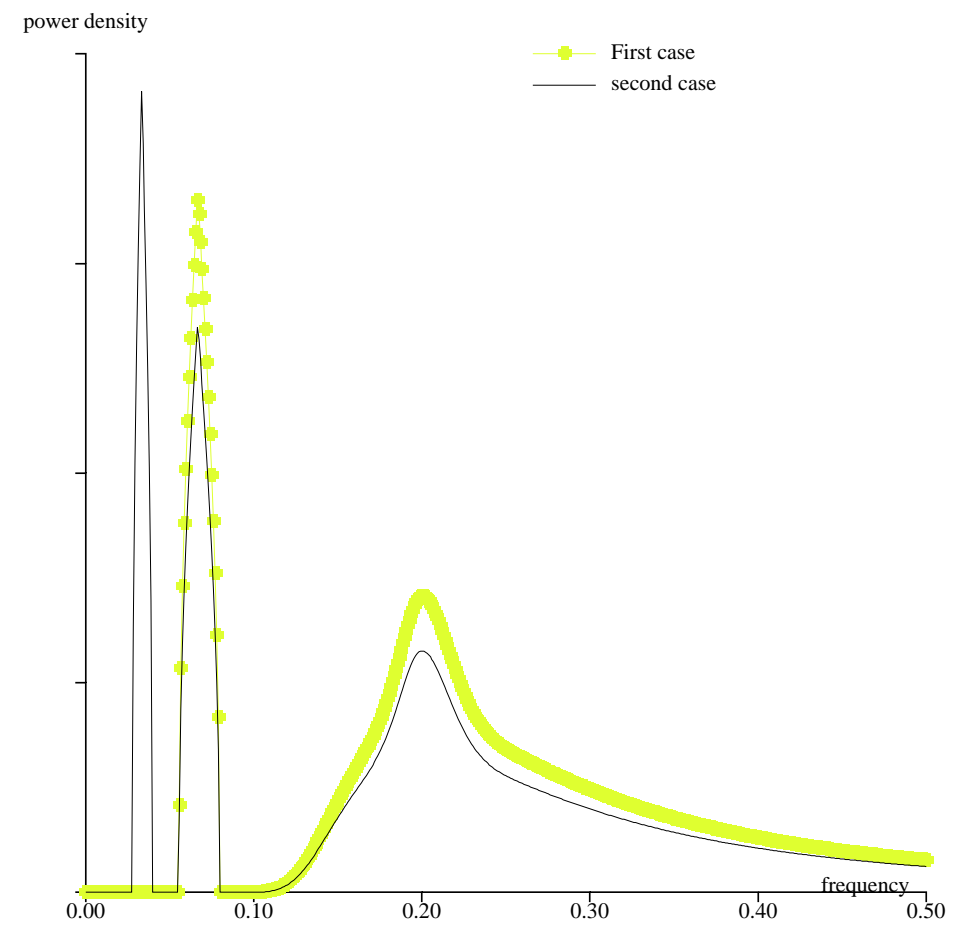

Fig. 4. Spectra of swell and wind sea used in the first application, and additionnal low-frequency mooring response in the second

5.2 Mooring plus quasi-static response to waves

Let us take the previous signal with the significant heights scaled down to 2.31 and add a low-frequency mooring response equivalent to 2.31 meter significant 


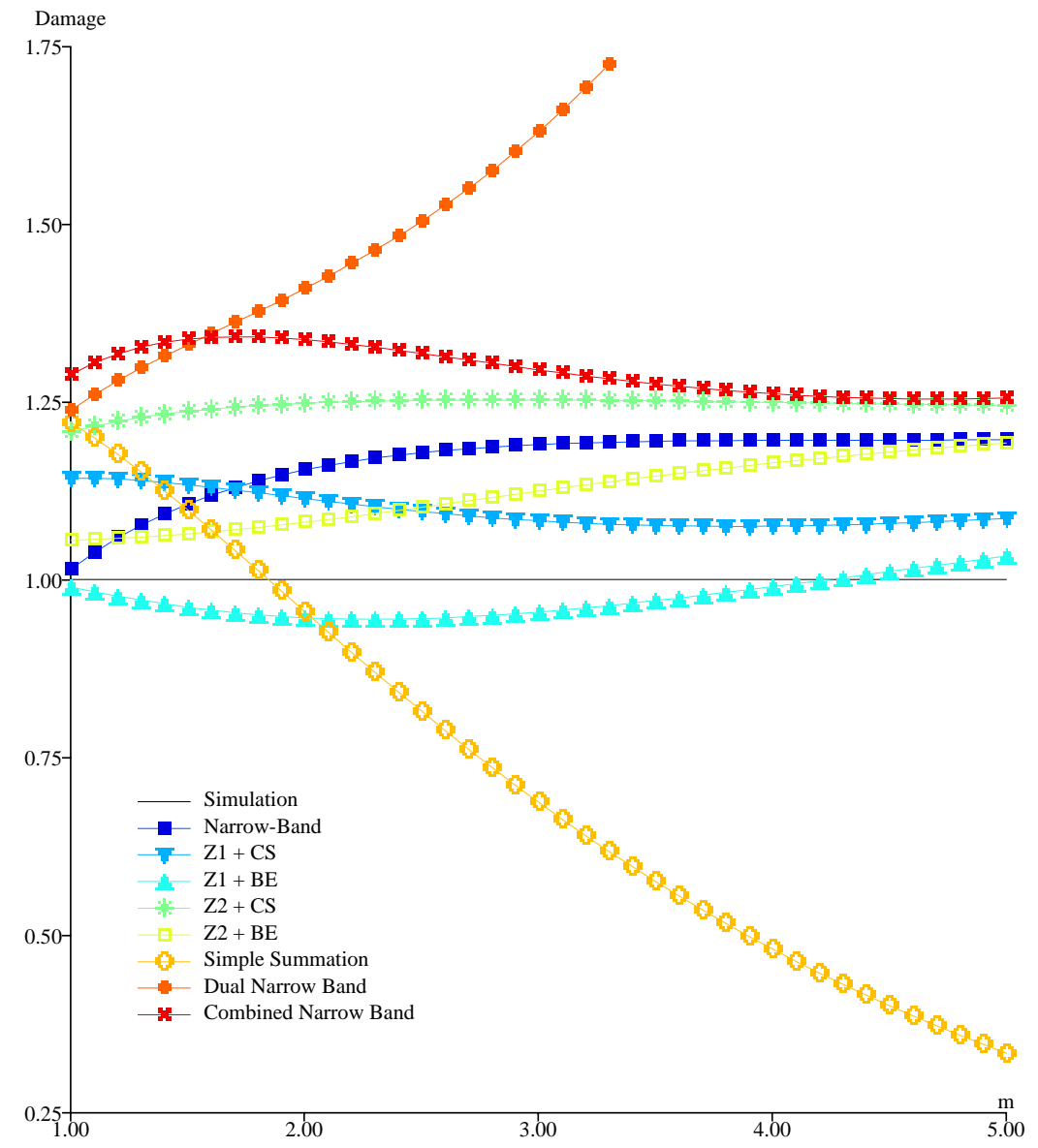

Fig. 5. Comparison of formulas for damage estimation, case 1

amplitude at 30 seconds with the same triangular shape as the swell. The previous spectrum now represents the high-frequency component, it is not especially narrow-banded. The corresponding values of the parameters are: $\alpha=0.8165, \zeta=0.1789, \beta=0.0929, \eta_{s}=0.4271$. 


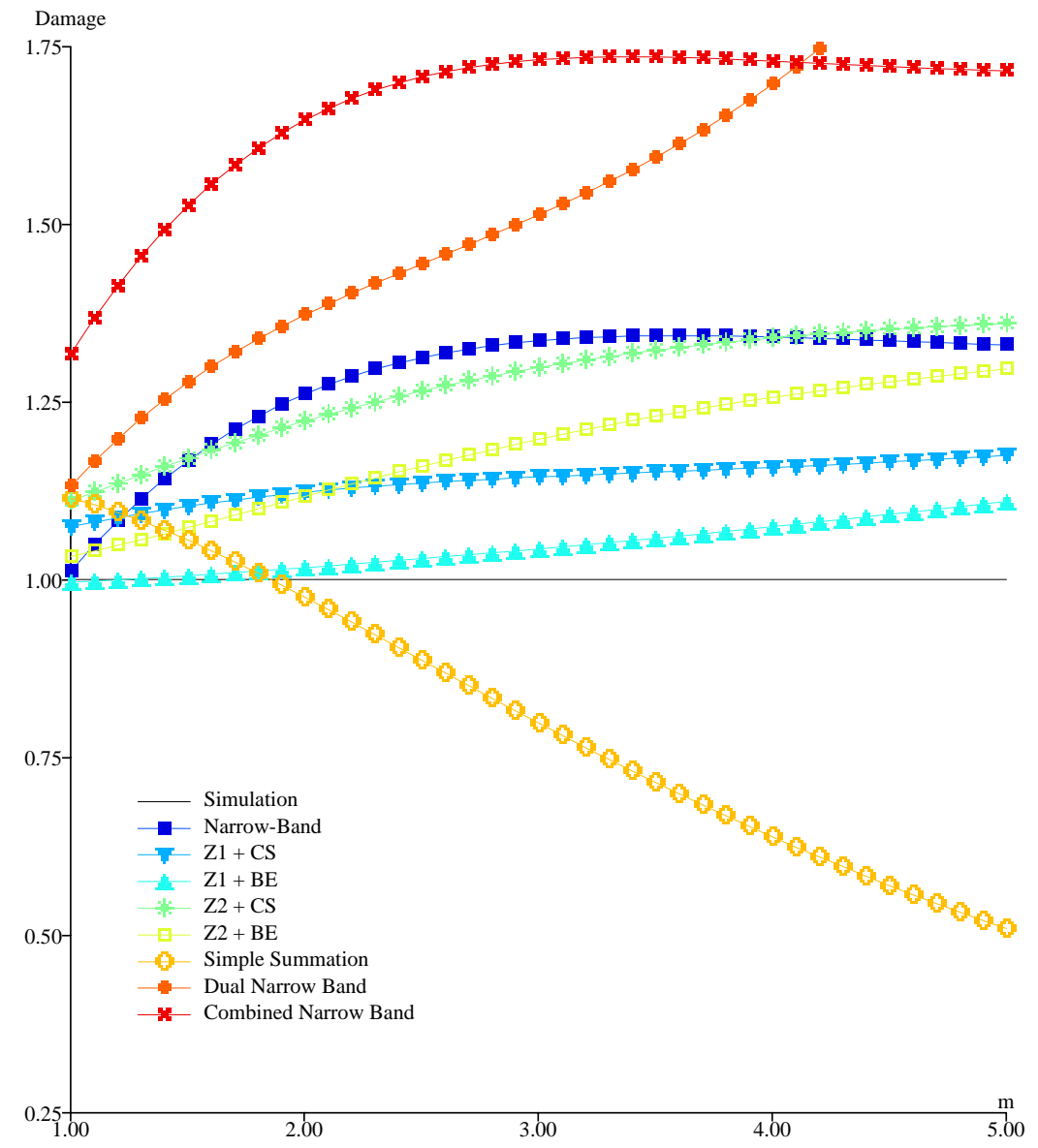

Fig. 6. Comparison of formulas for damage estimation, case 2

Combined narrow band and Dual narrow band methods are both definitely conservative. It can be seen that the two $Z_{1}$ approximations are slightly conservative, whereas the $Z_{2}$ ones are largely so, but still no more than the narrowband approximation. 
Resulting damage for the two cases is summarized in table 1.

Table 1

\begin{tabular}{|c|c|c|c|c|c|c|}
\hline & \multicolumn{3}{|c|}{ Case 1} & \multicolumn{3}{|c|}{ Case 2} \\
\hline$m$ & 1 & 3 & 5 & 1 & 3 & 5 \\
\hline$D_{\operatorname{simu}}$ & 1.000 & 1.000 & 1.000 & 1.000 & 1.000 & 1.000 \\
\hline$D_{\text {simuA }}$ & 0.480 & 0.154 & 0.051 & 0.651 & 0.258 & 0.102 \\
\hline$D_{\text {simu }}$ & 0.520 & 0.846 & 0.949 & 0.349 & 0.742 & 0.898 \\
\hline$D_{h}$ & 0.964 & 0.537 & 0.259 & 0.988 & 0.743 & 0.491 \\
\hline$D_{l}$ & 0.257 & 0.150 & 0.075 & 0.127 & 0.055 & 0.018 \\
\hline$D_{\text {unscaled } \mathcal{A}}$ & 0.788 & 0.439 & 0.212 & 0.897 & 0.674 & 0.446 \\
\hline$D_{c s \mathcal{A}}$ & 0.631 & 0.227 & 0.071 & 0.718 & 0.346 & 0.147 \\
\hline$D_{b e \mathcal{A}}$ & 0.480 & 0.099 & 0.018 & 0.640 & 0.245 & 0.083 \\
\hline$D_{z 1 \mathcal{B}}$ & 0.511 & 0.857 & 1.018 & 0.357 & 0.800 & 1.031 \\
\hline$D_{z 2 \mathcal{B}}$ & 0.568 & 1.015 & 1.165 & 0.391 & 0.947 & 1.207 \\
\hline$D_{b e+z 1}$ & 0.991 & 0.956 & 1.036 & 0.997 & 1.045 & 1.114 \\
\hline$D_{c s+z 1}$ & 1.141 & 1.084 & 1.089 & 1.075 & 1.146 & 1.178 \\
\hline$D_{b e+z 2}$ & 1.048 & 1.114 & 1.183 & 1.031 & 1.192 & 1.290 \\
\hline$D_{c s+z 2}$ & 1.199 & 1.242 & 1.236 & 1.109 & 1.293 & 1.354 \\
\hline$D_{N B}$ & 1.014 & 1.189 & 1.196 & 1.012 & 1.337 & 1.332 \\
\hline$D_{S S}$ & 1.221 & 0.687 & 0.334 & 1.115 & 0.798 & 0.509 \\
\hline$D_{D N B}$ & 1.225 & 1.594 & 2.580 & 1.118 & 1.389 & 1.899 \\
\hline$D_{C N B}$ & 1.271 & 1.256 & 1.222 & 1.301 & 1.589 & 1.617 \\
\hline
\end{tabular}

Damage $\left(\frac{m^{m}}{s}\right)$ for the combination of a swell and a wind sea (case 1) and an additional mooring response (case 2 )

\subsection{Iterative computation and two-slope $S-N$ curve}

Let us take the three-component case of 5.2, and assume that the S-N curve is a two-slope one, $m=3$ and $m=5$ with intersection point at $S=4$ (and thus $\left.K_{5}=16 K_{3}\right)$.

Table 2 summarizes the computations:

The final result is conservative by about $30 \%$. 


\section{Conclusions}

We have given two formulas to compute rainflow damage for composite signals made of one or several narrow-band low frequency components and of a higher frequency one, not necessarily narrow-band, from the individual properties and damages of each component. Those formulas are based on the mathematical definition of rainflow and on the theoretical properties of gaussian processes, the first one may be used to provide either best effort estimates, not garanteed to be conservative at all times, or conservative ones, and the second one conservative ones. Those formulas are free from the semi-empiricism that ruled many previous formulas for rainflow damage, see for instance Benasciutti 8 Tovo (2006). They allow for a wide-band high-frequency component, and thus for iterative addition of several narrow-band low-frequency ones, and for the introduction of two-slope S-N curves.

\section{Acknowledgements}

This paper owes much of its contents to more than a decade of discussions with Igor Rychlik over gaussian processes and rainflow counting.

\section{Appendix A: Distribution $\mathcal{M}$}

Let us drop the subscript of $\eta_{s}=\eta$ and denote $\epsilon=\sqrt{1-\eta_{s}^{2}}=\sqrt{1-\eta^{2}}$.

$\mathcal{M}$ is the distribution of the sum of 2 independent variables $\eta_{s} R$ and $\epsilon G$, where $R$ and $G$ are normalized Rayleigh and Gaussian distributed. Thus,

$$
\begin{aligned}
& m_{\mathcal{M}}(x=v)=\int_{0}^{\infty} p_{\mathcal{R}}(\eta R=u) p_{\mathcal{G}}(\epsilon G=v-u) d u \\
& m_{\mathcal{M}}(x=v)=\int_{0}^{\infty} \frac{u}{\eta^{2}} e^{-\frac{u^{2}}{2 \eta^{2}}} \frac{1}{\epsilon \sqrt{2 \pi}} e^{-\frac{(v-u)^{2}}{2 \epsilon^{2}}} d u \\
& m_{\mathcal{M}}(x=v)=\frac{\epsilon}{\sqrt{2 \pi}} e^{-\frac{v^{2}}{2 \epsilon^{2}}}+\frac{\eta}{2} v e^{-\frac{v^{2}}{2}} \operatorname{erfc}\left(-\frac{\eta}{\epsilon} \frac{v}{\sqrt{2}}\right) \\
& m_{\mathcal{M}}(x=v)=\frac{1}{\sqrt{2}}\left(\frac{\sqrt{\left(1-\eta^{2}\right)}}{\sqrt{\pi}} e^{-\frac{v^{2}}{2} \frac{1}{\left(1-\eta^{2}\right)}}+\eta \frac{v}{\sqrt{2}} e^{-\frac{v^{2}}{2}} \operatorname{erfc}\left(-\frac{\eta}{\sqrt{\left(1-\eta^{2}\right)}} \frac{v}{\sqrt{2}}\right)\right)
\end{aligned}
$$

The cumulative distribution is obtained as: 


$$
\begin{aligned}
& \mathcal{M}(x \leq v)=\frac{\epsilon}{\sqrt{2 \pi}} \int_{-\infty}^{v} e^{-\frac{u^{2}}{2 \epsilon^{2}}} d u+\frac{\eta}{2} \int_{-\infty}^{v} u e^{-\frac{u^{2}}{2}} \operatorname{erfc}\left(-\frac{u \eta}{\epsilon \sqrt{2}}\right) d u \\
& \mathcal{M}(x \leq v)=\frac{1}{2}\left(\operatorname{erfc}\left(-\frac{1}{\sqrt{1-\eta^{2}}} \frac{v}{\sqrt{2}}\right)-\eta e^{-\left(\frac{v}{\sqrt{2}}\right)^{2}} \operatorname{erfc}\left(-\frac{\eta}{\sqrt{1-\eta^{2}}} \frac{v}{\sqrt{2}}\right)\right)
\end{aligned}
$$

\section{Appendix B: Distributions $\mathcal{Y}_{1}$ and $\mathcal{Y}_{2}$}

$$
\begin{aligned}
\mathcal{Y}_{1}(x)= & \mathcal{M}^{\frac{N_{h}}{N_{l}}}(x)=\mathcal{M}^{\frac{1}{\beta}}(x) \\
y_{1}(x) d x= & \frac{1}{\beta} m_{\mathcal{M}}(x) \mathcal{M}^{\frac{1}{\beta}-1}(x) d x \\
y_{1}(x) d x= & \frac{1}{2^{\frac{1}{\beta}} \beta}\left(\frac{\sqrt{2\left(1-\eta^{2}\right)}}{\sqrt{\pi}} e^{-\frac{x^{2}}{2\left(1-\eta^{2}\right)}}+\eta x e^{-\frac{x^{2}}{2}} \operatorname{erfc}\left(-\frac{x \eta}{\sqrt{2\left(1-\eta^{2}\right)}}\right)\right) \\
& \left.\times\left(\operatorname{erfc}\left(-\frac{1}{\sqrt{1-\eta^{2}}} \frac{x}{\sqrt{2}}\right)-\eta e^{-\left(\frac{x}{\sqrt{2}}\right)^{2}} \operatorname{erfc}\left(-\frac{\eta}{\sqrt{1-\eta^{2}}} \frac{x}{\sqrt{2}}\right)\right)\right)^{\frac{1}{\beta}-1} d x \\
z_{1}(\beta, \eta, t) d t= & \frac{2\left(1-\eta^{2}\right)}{2^{\frac{1}{\beta}} \beta}\left(\frac{e^{-t^{2}}}{\sqrt{\pi}}+\eta t e^{-\left(1-\eta^{2}\right) t^{2}} \operatorname{erfc}(-\eta t)\right) \\
& \times\left(\operatorname{erfc}(-t)-\eta e^{-\left(1-\eta^{2}\right) t^{2}} \operatorname{erfc}(-\eta t)\right)^{\frac{1}{\beta}-1} d t \\
Z_{1}(m, \beta, \eta)= & \frac{1}{\Gamma\left(\frac{m}{2}+1\right)} \int_{0}^{\infty} t^{m} z_{1}(\beta, \eta, t) d t
\end{aligned}
$$

$\delta_{2}$ is defined as the solution of:

$$
\begin{aligned}
& \operatorname{erfc}\left(-\frac{1}{\sqrt{1-\eta^{2}}} \frac{\delta_{2}}{\sqrt{2}}\right)-\eta e^{-\left(\frac{\delta_{2}}{\sqrt{2}}\right)^{2}} \operatorname{erfc}\left(-\frac{\eta}{\sqrt{1-\eta^{2}}} \frac{\delta_{2}}{\sqrt{2}}\right)=2(1-\beta) \\
& \mathcal{Y}_{2}(x)=1-\frac{1}{\beta}(1-\mathcal{M}(x)), x \geq \delta_{2} \\
& y_{2}(x) d x=\frac{\sqrt{2\left(1-\eta^{2}\right)}}{2 \beta \sqrt{\pi}} e^{-\frac{x^{2}}{2\left(1-\eta^{2}\right)}} d x+\frac{\eta}{\beta} \frac{x}{2} e^{-\frac{x^{2}}{2}} \operatorname{erfc}\left(-\frac{x \eta}{\sqrt{2\left(1-\eta^{2}\right)}}\right) d x, x \geq \delta_{2}
\end{aligned}
$$

Let $\xi_{2}=\frac{\delta_{2}}{\sqrt{2} \sqrt{1-\eta^{2}}}$, thus verifying: 


$$
\begin{aligned}
2-\operatorname{erfc}\left(-\xi_{2}\right) & =2 \beta-\eta e^{-\left(1-\eta^{2}\right) \xi_{2}^{2}} \operatorname{erfc}\left(-\eta \xi_{2}\right) \\
z_{2}(\beta, \eta, t) d t & =\frac{\left(1-\eta^{2}\right)}{\beta \sqrt{\pi}} e^{-t^{2}} d t+\frac{\eta}{\beta}\left(1-\eta^{2}\right) t e^{-\left(1-\eta^{2}\right) t^{2}} \operatorname{erfc}(-\eta t) d t, t \geq \xi_{2} \\
& =\frac{\left(1-\eta^{2}\right) e^{-t^{2}}}{\beta}\left(\frac{1}{\sqrt{\pi}}+\eta t e^{\eta^{2} t^{2}} \operatorname{erf} c(-\eta t)\right) d t, t \geq \xi_{2} \\
Z_{2}(m, \beta, \eta, x) & =\frac{1}{\Gamma\left(\frac{m}{2}+1\right)} \int_{x}^{\infty} t^{m} z_{2}(\beta, \eta, t) d t
\end{aligned}
$$

\section{References}

Benasciutti, D. \& Tovo, R., 2006, Comparison of Spectral Methods for Fatigue Analysis of Broad-Band Random Processes, Probabilistic Engineering Mechanics, Vol. 21, 287-299.

Brodtkorb, P.A., Lingren, G. Rychlik, I., Rydén, J. \& Sjö, E., 2000, WAFO: a Matlab toolbox for analysis of random waves and loads, Proc. 10th International Offshore and Polar Engineering Conference, ISOPE, 3, 343-350.

Huang, W., Moan, T., 2006, Fatigue under Combined High and Low Frequency Loads, Proc. 25th Int. Conference on Offshore Mechanics and Arctic Engineering, OMAE2006-92247.

ISSC, P.A. Frieze \& R.A. Shenoi eds., Report of Technical Committee III.2 Fatigue and Fracture, Proc. 16th ISSC, Vol. 1, 439-520.

Jiao, G., Moan, T., 1990, Probabilistic analysis of fatigue due to Gaussian load processes, Probabilistic Engineering Mechanics, Vol. 5, No. 2, 76-83

Lie, H. \& Fylling, J., 1994, Evaluation of Methods for Fatigue Analysis of Offshore Mooring Lines, Proc. 10th Offshore South East Asia Conf., OSEA94007.

Lotsberg, I., 2005, Background for Revision of DNV-RP-C203 Fatigue Analysis of Offshore Steel Structure, Proc. 24th Int. Conference on Offshore Mechanics and Arctic Engineering, OMAE2005-67549.

Matsuishi, M., Endo, T., 1968, Fatigue of Metals Subjected to Varying Stress, Japan Society of Mechanical Engineers, Jukvoka, Japan.

Rychlik, I., 1987, A new definition of the rainflow counting method, Int. J. Fatigue 9, 119-121. 


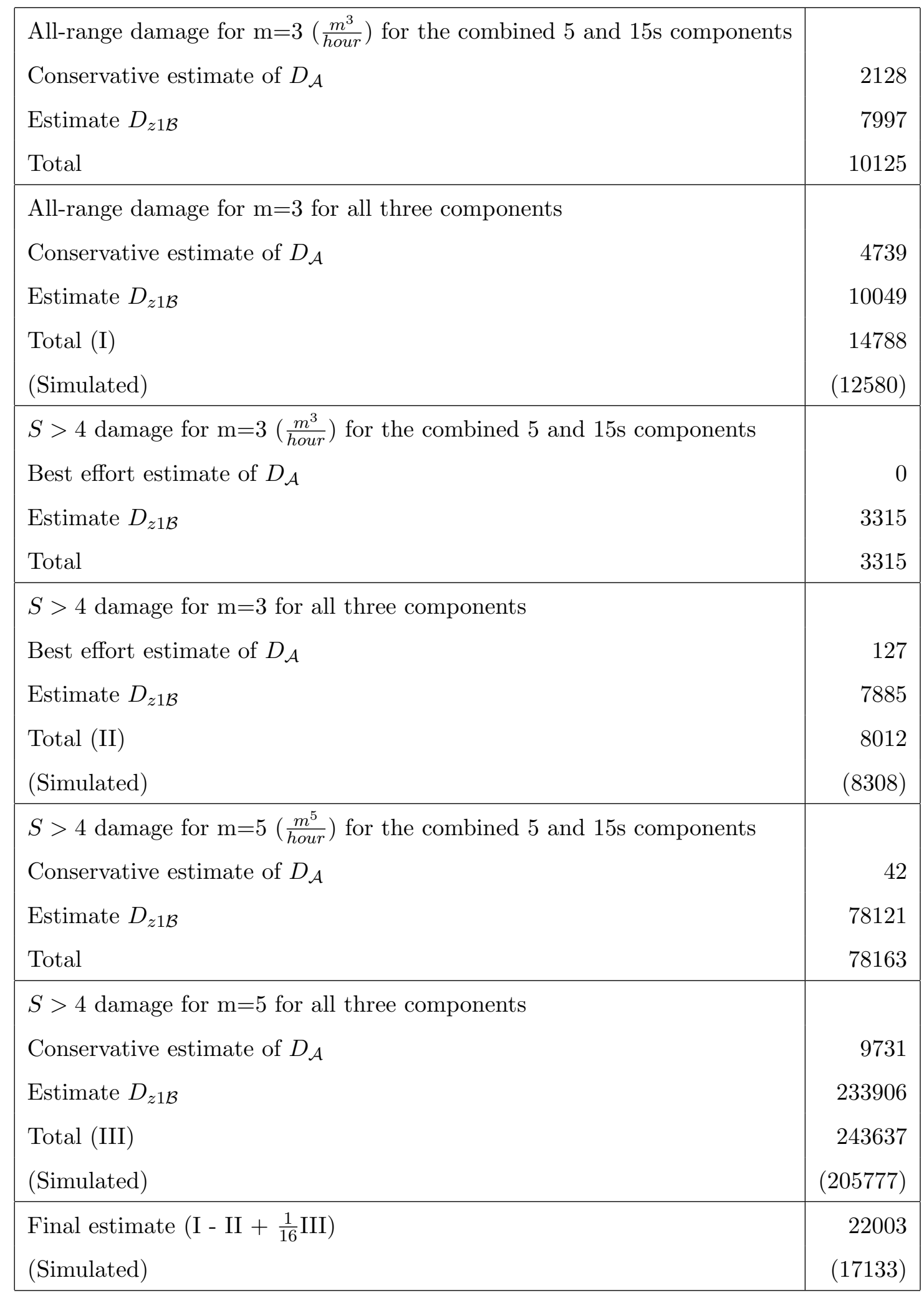

Table 2

Computation steps for iterative two-slope case 